\title{
Meta-analysis on risk stratification of malignant ventricular tachyarrhythmic events in arrhythmogenic right ventricular cardiomyopathy
}

\author{
Young-Eun Roh, Hyun Ji Jang, Min-Jung Cho \\ Medical Research Institute of Pusan National University Hospital, Pusan National University School of Medicine, Busan, Korea
}

Background: Arrhythmogenic right ventricular cardiomyopathy (ARVC) is a cardiomyopathy characterized by predominant right ventricular fibro-fatty replacement, right ventricular dysfunction and ventricular arrhythmias. It is a rare but important cause of sudden cardiac death in children and young adults. A meta-analysis on risk stratification of major ventricular tachyarrhythmic events indicating the need for implantable cardioverter defibrillator therapy in ARVC was performed.

Methods: The pubmed database was searched from its inception to May 2015. Of the 433 citations identified, 12 were included in this meta-analysis. Data regarding major ventricular tachyarrhythmic events were retrieved in 817 subjects from the studies. For the variables, a combined odds ratio (OR) was calculated using a fixed-effects meta-analysis.

Results: Extensive right ventricular dysfunction (OR, 2.44), ventricular late potential (OR, 1.66), inducible ventricular tachyarrhythmia during electrophysiology study (OR, 3.67), non-sustained ventricular tachycardia (OR, 3.78), and history of fatal event/sustained VT (OR, 5.66) identified as significant risk factors $(p<0.0001)$.

Conclusion: This meta-analysis shows that extensive right ventricular dysfunction, ventricular late potential, inducible ventricular tachyarrhythmia during electrophysiological study, non-sustained ventricular tachycardia, and history of sustained ventricular tachycardia/fibrillation are consistently reported risk factors of major ventricular tachyarrhythmic events indicating implantable cardioverter defibrillator therapy in patients with ARVC.

Keywords: Arrhythmogenic right ventricular cardiomyopathy; Defibrillator; Sudden death; Ventricular tachyarrhythmia; Meta-analysis

\section{INTRODUCTION}

Arrhythmogenic right ventricular cardiomyopathy (ARVC) is a progressive cardiomyopathy characterized by fibro-fatty replacement of the right ventricle, predominant right ventricular dilatation and dysfunction, and/or ventricular tachyarr-

Received: October 12, 2017, Revised: October 24, 2017 Accepted: October 25, 2017

Corresponding Author: Min-Jung Cho, Division of Pediatric Cardiology, Department of Pediatrics, Pusan National University Hospital, 179, Gudeok-ro, Seo-gu, Busan 49241, Korea

Tel: +82-51-240-7800, Fax: +82-51-248-6205

E-mail: mjchomd@gmail.com hythmias. It is a rare but important cause of sudden cardiac death in children and young adults [1]. Furthermore, it has been reported to be an important cause of sports-related sudden cardiac arrest [1]. Although implantable cardioverter defibrillator (ICD) therapy is necessary in the secondary prevention after survived cardiac arrest, or sustained ventricular tachyarrhythmias in the patients [2,3], its role for the primary prevention in asymptomatic patients with ARVC with no history of sustained tachyarrhythmias or cardiac arrest remains unclear.

The aim of this meta-analysis was to identify patients with ARVC at a high risk of ventricular tachyarrhythmias, who might benefit from ICD therapy, via a systematic review of

Copyright (C) 2017 Yeungnam University College of Medicine

This is an Open Access article distributed under the terms of the Creative Commons Attribution Non-Commercial License (http://creativecommons.org/licenses/by-nc/4.0/) which permits unrestricted non-commercial use, distribution, and reproduction in any medium, provided the original work is properly cited. 
the literature and to analyze the predictive value of currently evaluated risk factors via a meta-analysis.

\section{MATERIALS AND METHODS}

\section{Search strategy and study selection}

We performed a computerized search of the literature in the PubMed database (from the time of inception to May 2015) to identify eligible studies focusing on the identification of patients with the ARVC at risk of ventricular tachyarrhythmia or sudden cardiac death, to whom the ICD therapy might be beneficial. We used a combination of the following keywords: "Arrhythmogenic Right Ventricular Dysplasia", "defibrillator", "ventricular tachycardia" and "sudden death". We subsequently reviewed the reference lists of all primary identified studies to find any relevant publications that were not named in the database search. Case reports and review articles were excluded. The search was limited to human studies and those in the English language. The titles and abstracts of all articles were reviewed and rejected after initial screening according to the following exclusion criteria: (1) studies on all cause of cardiac mortality, including heart failure and transplantation, (2) studies on atrial arrhythmias, ablation, and mapping, (3) studies with incomplete data, and (4) studies with overlapping data or apparent serial reporting of a particular patient cohort. There were no restrictions in the sample size or duration of follow-up. Two authors (Roh and Jang) independently screened the titles and abstracts of all the citations. Disagreements were resolved by discussing with Cho. Full-text articles were retrieved for a detailed review and then rescreened.

\section{Data extraction}

The following data were extracted: (1) year of publication, (2) study design, (3) patient demographics including sample size, age, sex, duration of follow up, family history, number of patients with ICD, and detailed information regarding candidate risk factors, and (4) relevant outcomes: occurrence of sustained ventricular tachyarrhythmias, appropriate device therapies, or arrhythmic death as determined by the individual study methods.

\section{Data synthesis and analysis}

The analyses were performed using the Review Manager version 5.1.0 software (Nordic Cochrane Centre, Cochrane Collaboration, Copenhagen, Denmark). The results were expressed as odds ratios (ORs) with 95\% confidence intervals (CIs) for each study. Heterogeneity was assessed using the Cochran Q-test ( $p<0.10$ was considered significant), and I2 statistics (I2 $>56 \%$ was considered as an indicator of significant heterogeneity) were calculated to estimate the proportion of variation attributable to heterogeneity across the studies. The studies that were homogenous for an outcome were analyzed using the Mantel-Haenszel fixed-effects model. In cases where evidence of heterogeneity was noted, a random-effects model was used. Publication bias was assessed using funnel plots. Statistical significance was defined as a two-sided $p$ value $<0.05$ for all tests except those for heterogeneity.

\section{RESULTS}

\section{Identified studies}

Four hundred thirty-three studies were identified in the primary literature search. After screening the titles and abstracts, 69 relevant studies were retrieved for detailed evaluation. Fifty-seven of them were further excluded. The results of the literature search and exclusion reasons are shown in Figure 1.

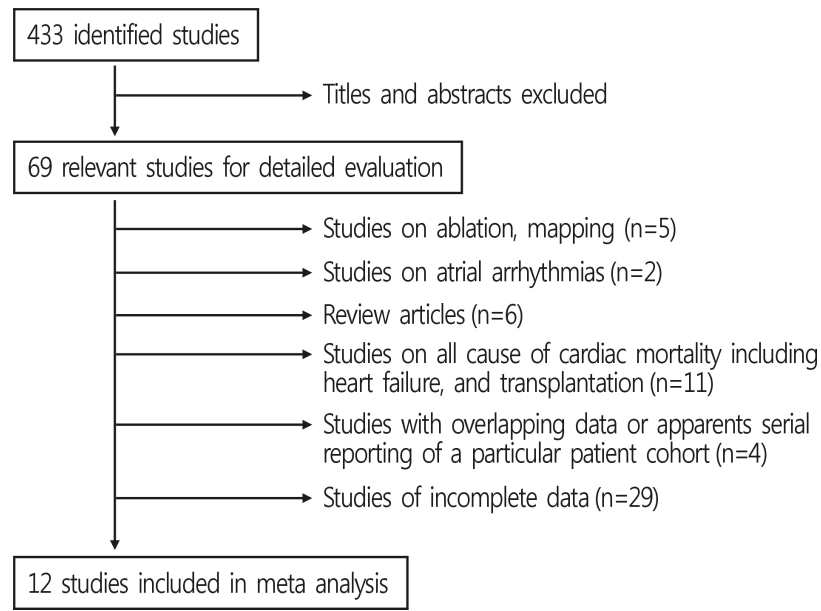

Fig. 1. Study selection flow diagram. 
A total of 817 subjects with ARVC from 12 studies were finally included in our meta-analysis [4-15]. The characteristics of the individual studies are described in Table 1. The ages of the study patients varied from 2 to 78 years. The mean follow-up period of the study ranged from 19 to 80 months. The prevalence of appropriate ICD therapy or lethal arrhythmic events during follow up varied from $17 \%$ to $69 \%$.

\section{Identified candidate risk factors}

A total of 19 candidate risk factors have been evaluated in one or more studies (Table 2). Nine of the 19 variables were available for the meta-analysis; extensive right ventricular dysfunction, left ventricular involvement, ventricular late potential on signal averaged electrocardiography (SAECG), T-wave inversion on the right precordial leads on electrocardiography, history of syncope, inducible ventricular tachyarrhythmias during electrophysiologic study, frequent ventricular premature beats on 24-hour Holter monitoring, nonsustained ventricular tachyarrhythmias, and history of sustained ventricular tachyarrhythmias (Fig. 2, Table 3).

Extensive right ventricular dysfunction was investigated in seven studies. Three of the sevenstudies were available for the subgroup analysis, which demonstrated that extensive right ventricular dysfunction in ARVC predicted an increased risk of future ventricular tachyarrhythmic events during the follow-up period $(\mathrm{OR}=2.44,95 \% \mathrm{CI}=1.02-5.82, \mathrm{z}=2.01$, $p=0.04)$. Left ventricular involvement has also been evaluated in most of included studies (11/12 studies); we did not find any statistical correlation between left ventricular involvement and risk of future tachyarrythmic events in the subgroup analysis of five feasible studies $(\mathrm{OR}=1.80,95 \% \mathrm{CI}=$ 1.00-3.24, $\mathrm{z}=1.96, p=0.05$ ).

Eight studies assessed whether ventricular late potential is a predictor of ventricular arrhythmias; existence of ventricular late potential might be a weak, but significant predictive factor of ventricular arrhythmias according to the subgroup analysis of five studies $(\mathrm{OR}=1.66,95 \% \mathrm{CI}=1.03-2.68, \mathrm{z}=$ 2.08, $p=0.04$ ). T-wave inversion on the right precordial leads was investigated in five studies, and four studies were available for the subgroup analysis. There was no significant association between T-wave inversion on the right precordial leads and major ventricular tachyarrhythmic events ( $\mathrm{OR}=1.79,95 \%$ $\mathrm{CI}=0.97-3.31, \mathrm{z}=1.86, p=0.06$ ).

Data regarding the history of syncope for the risk of ventricular tachyarrhythmias were retrieved from eight articles. A history of syncope was not a significant predictor of life-threatening ventricular arrhythmias according to this subgroup analysis $(\mathrm{OR}=1.41,95 \% \mathrm{CI}=0.65-3.05, \mathrm{z}=0.86, p=0.39)$.

Inducible ventricular tachyarrhythmias during electrophysiologic study were investigated in seven articles; five of them were available for the subgroup analysis. These studies showed higher risks of future ventricular tachyarrhythmic events in patients ( $\mathrm{OR}=3.67,95 \% \mathrm{CI}=1.58-8.49, \mathrm{z}=3.03, p=0.0002)$. Frequent ventricular premature beats on 24-hour Holter monitoring were evaluated as predictors of ventricular tachya-

Table 1. Main characteristics of the selected studies

\begin{tabular}{|c|c|c|c|c|c|c|c|c|c|c|c|c|}
\hline & $\begin{array}{l}\text { Corrado } \\
2003[4]\end{array}$ & $\begin{array}{l}\text { Wichter } \\
2004[5]\end{array}$ & $\begin{array}{c}\text { Piccini } \\
2005 \text { [6] }\end{array}$ & $\begin{array}{l}\text { Pezawas } \\
2006[7]\end{array}$ & $\begin{array}{l}\text { Corrado } \\
2010[8]\end{array}$ & $\begin{array}{l}\text { Bhonsale } \\
2011 \text { [9] }\end{array}$ & $\begin{array}{c}\text { Schuler } \\
2012[10]\end{array}$ & $\begin{array}{c}\text { Battipaglia } \\
2012 \text { [11] }\end{array}$ & $\begin{array}{l}\text { Santangeli } \\
2012[12]\end{array}$ & $\begin{array}{c}\text { Canpolat } \\
2013[13]\end{array}$ & $\begin{array}{c}\text { Link } \\
2014[14]\end{array}$ & $\begin{array}{l}\text { Ruiz-Salas } \\
2014 \text { [15] }\end{array}$ \\
\hline Cohort & Italy, USA & Germany & USA & Austria & $\begin{array}{l}\text { Italy, UK, } \\
\text { Germany, USA }\end{array}$ & USA & Switzerland & Italy & $\begin{array}{l}\text { Italy, } \\
\text { USA }\end{array}$ & Turkey & USA & Spain \\
\hline $\mathrm{n}$ & 132 & 60 & 67 & 34 & 106 & 84 & 26 & 30 & 32 & 78 & 137 & 31 \\
\hline Age (yr) & $40 \pm 15(15-72)$ & $43 \pm 16(1470)$ & $36 \pm 14(2-78)$ & $49 \pm 12(1468)$ & $35.6 \pm 18(16-65)$ & $31.9 \pm 11(11-59)$ & median 40 & $45.4 \pm 18$ & $48 \pm 15$ & $31.2 \pm 11$ & $40 \pm 14$ & $47.3 \pm 17$ \\
\hline$\%$ Male & 70 & 81 & 52 & 61 & 67 & 64 & 80 & 56 & NA & 65.4 & 68.6 & 77.4 \\
\hline $\begin{array}{l}\text { \% FHx. of } \\
\text { RVD/SD }\end{array}$ & 3 & 15 & 22 & NA & 46 & 17 & 15.3 & 26 & NA & 26.9 & NA & 16.13 \\
\hline Follow up (mo) & $39 \pm 25$ & $80 \pm 43$ & $52 \pm 34$ & $78 \pm 28$ & $58 \pm 35$ & $56 \pm 40$ & median 120 & $19 \pm 7$ & $25 \pm 7$ & $38 \pm 14$ & $39 \pm 20$ & $73 \pm 64$ \\
\hline $\begin{array}{l}\% \text { of ICD } \\
\text { implantation (n) }\end{array}$ & 100 (132) & $100(60)$ & $100(67)$ & $47(16)$ & $100(106)$ & $100(84)$ & $100(26)$ & $50(15)$ & $100(32)$ & $59(46)$ & $78(108)$ & $100(31)$ \\
\hline $\begin{array}{l}\% \text { of prophylactic } \\
\text { ICD in total ICD } \\
\text { (n) }\end{array}$ & $28(37)$ & $7(4)$ & $41(28)$ & 0 & $100(106)$ & $100(84)$ & $3(1)$ & $100(15)$ & $100(32)$ & $45.6(21)$ & NA & $19(6)$ \\
\hline $\begin{array}{l}\% \text { of appropriate } \\
\text { ICD Tx or lethal } \\
\text { events }(n)\end{array}$ & $48(64)$ & $69(41)$ & $66(59)$ & $34(12)$ & $40(42)$ & $48(40)$ & $46(12)$ & $17(5)$ & $38(12)$ & $50(39)$ & $44(48)$ & $61(19)$ \\
\hline
\end{tabular}

ARVD/SD, arrhythmogenic right ventricular dysplasia/sudden death; ICD, implantable cardioverter-defibrillator. 
rrhythmias in seven studies. Meta-analysis was available in five studies. Combining the five studies available for the metaanalysis yielded an OR of $1.24(95 \% \mathrm{CI}=0.75-2.07)$, indicating no significant association with an increased risk of ventricular tachyarrhythmias $(\mathrm{z}=0.84, p=0.40)$. Non-sustained ventricular tachyarrhythmia was investigated in six studies, with four studies available for the meta-analysis. Non-sustained ventricular tachyarrhythmia was identified as a significant predictor of ventricular tachyarrhythmias and appropriate $\mathrm{ICD}$ therapy $(\mathrm{OR}=3.78,95 \% \mathrm{CI}=2.08-6.85, \mathrm{z}=4.38$, $p<0.0001)$.

Table 2. Identified candidate risk factors

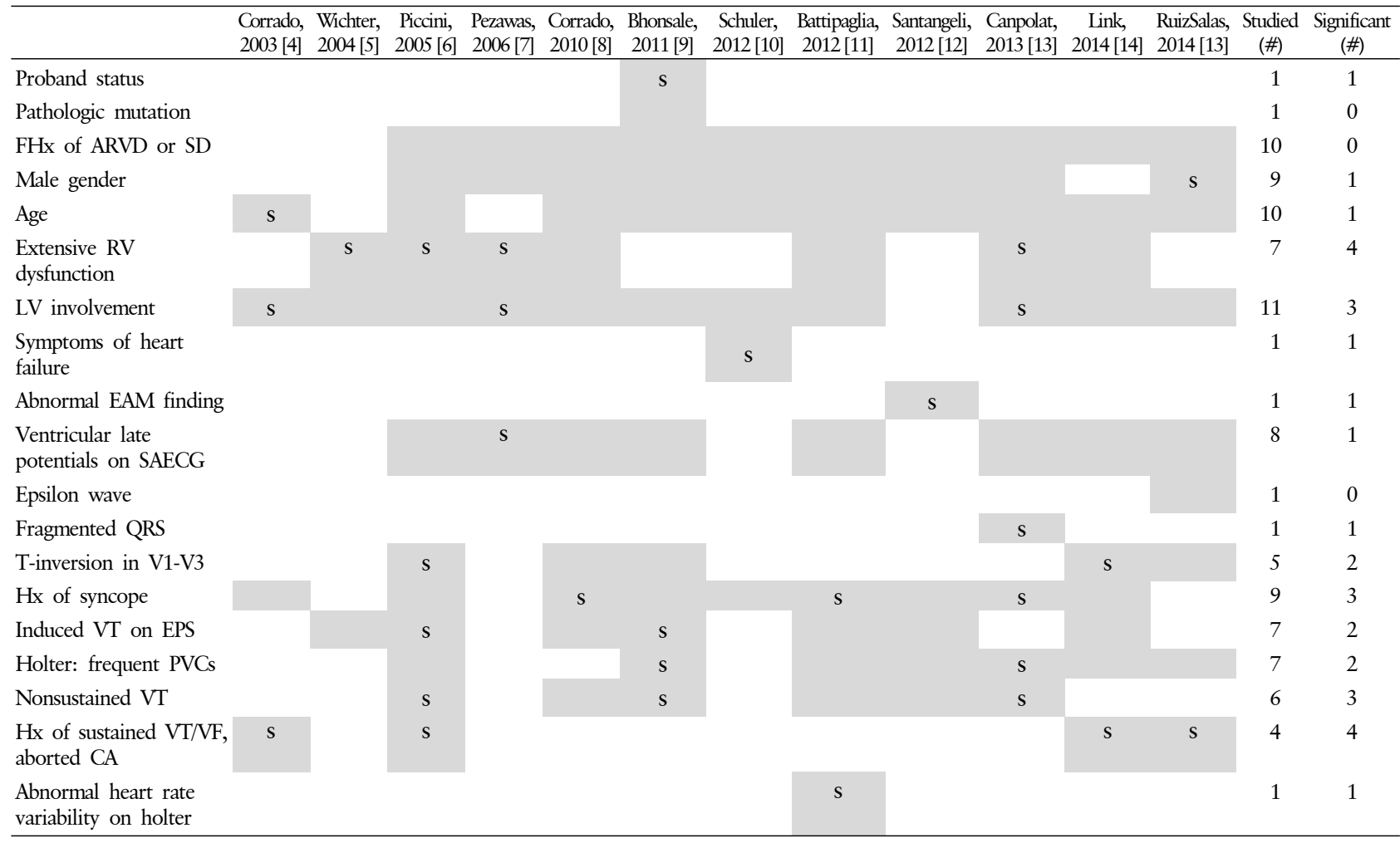

ARVD, arrhythmogenic right ventricular dysplasia; SD, sudden death; RV, right ventricle; LV, left ventricle; EAM, electroanatomic mapping; SAECG, signal averaged Electrocardiography; VT, ventricular tachycardia; EPS, electrophysiologic study; PVC, premature ventricular complex; VF, ventricular fibrillation; CA, cardiac arrest.

Shaded indicates that sufficient analysis results were provided in the paper to be included in meta-analysis; s, significant association with risk.

Table 3. Combined risk of candidate risk factors

\begin{tabular}{lllll}
\hline Candicate risk factor & $\mathrm{n}$ & OR & CI & $p$-value \\
\hline Extensive RV dysfunction & 200 & 2.44 & $1.02-5.82$ & 0.04 \\
LV involvement & 313 & 1.8 & $1.00-3.24$ & 0.05 \\
Ventricular late potential & 364 & 1.66 & $1.03-2.68$ & 0.04 \\
T-wave inversion on V1-3 & 285 & 1.76 & $0.97-3.31$ & 0.06 \\
Hx of syncope & 585 & 1.41 & $0.65-3.05$ & 0.39 \\
Inducible VT/VF during EPS & 344 & 3.67 & $1.58-8.49$ & 0.002 \\
Frequent PVCs on holter & 300 & 1.24 & $0.75-2.07$ & 0.4 \\
Nonsustained VT & 248 & 3.78 & $2.08-6.85$ & $<0.00001$ \\
Fatal event/sustained VT & 452 & 5.66 & $3.36-9.53$ & $<0.00001$
\end{tabular}

OR, odds ratio; CI, confidence interval; RV, right ventricle; LV, left ventricle; Hx, history; VT, ventricular tachycardia; VF, ventricular fibrillation; EPS, electrophysiologic study; PVC, premature ventricular complex. 
Young-Eun Roh et al.

\begin{abstract}
a. Extensive right ventricular dysfunction
\end{abstract}

b. LV involvement

d. $T$ - inversion on lead V1-3

e. History of syncope

f. Inducible VTNF during EPS

g. Frequent PVCs on Holter

h. Nonsustained VT

i. Fatal event / sustained VT
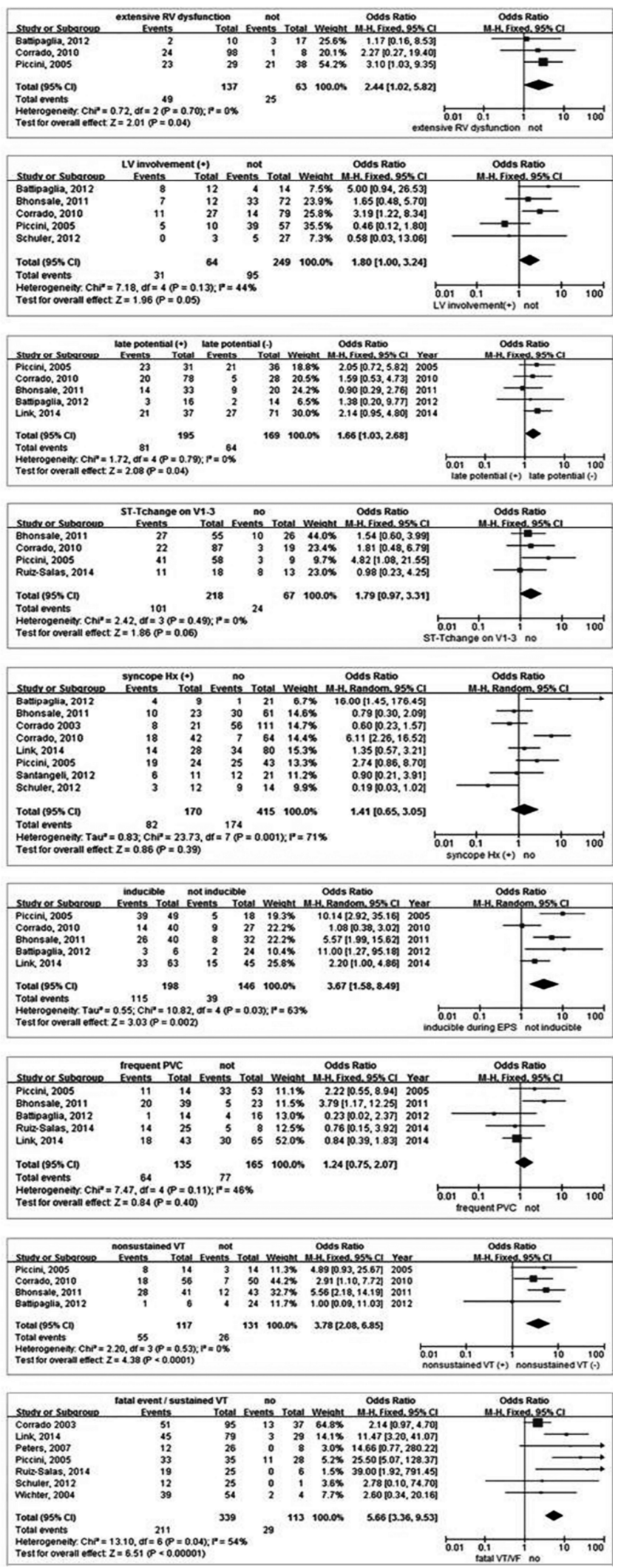

Fig. 2. Forest plots showing. RV, right ventricular; LV, left ventricular; VT, ventricular tachycardia; VF, ventricular fibrillation; PVCs, premature ventricular complexes. 


\section{DISCUSSION}

Although ICD therapy is widely accepted in the termination of life-threatening tachyarrhythmias in ARVC, its role for the primary prevention of ventricular tachyarrhythmias and sudden cardiac death in asymptomatic ARVC patients remains controversial, as not all patients with ARVC are at risk for such events [16]. Further, frequent device-related complications and inappropriate interventions mostly in young patients who are expected to live for many years with the device make the decision-making process more complicated. Some of the most studied risk factors for ventricular tachyarrhythmias/ICD therapy include extensive right ventricular dysfunction, coexisting left ventricular involvement, unexplained syncope, induction of ventricular tachyarrhythmias during electrophysiological testing, non-sustained ventricular tachyarrhythmias on noninvasive monitoring, male sex, familial history of sudden death, and young age at presentation $[4,7-11,17,18]$. In our meta-analysis, the pooled data have confirmed that severe right ventricular involvement, inducible ventricular tachyarrhythmias during electrophysiologic study, and nonsustained ventricular tachyarrhythmias on 24-hour Holter monitoring and/or exercise testing are significant predictors of ventricular tachyarrhythmias and ICD therapy in asymptomatic patients with ARVC.

Pathologically, ARVC is characterized by a fibro-fatty replacement primarily affecting the right ventricular myocardium [19-21], which is responsible for regional wall motion abnormalities and/or global right ventricular dysfunction [20,22]. Extensive right ventricular dysfunction detected on echocardiography or magnetic resonance imaging could be a meaningful risk factor of ventricular tachyarrhythmias, usually originating from the right ventricle. Late potentials detected on SAECG represent delayed depolarization of the ventricular myocardium; thus they can be considered another marker of right ventricular problems $[23,24]$. In our meta-analysis, a significant association was observed between late potentials and occurrence of tachyarrhythmias in the patients with ARVC. The presence of $\mathrm{T}$-wave inversion in $\mathrm{V} 1-\mathrm{V} 3$ or premature ventricular complexes of the left bundle branch block morphology on 12-lead electrocardiography could also be the first alarming signs of right ventricular change [25]; however, their values in identifying patients at risk of ventricular tachyarrhythmias/ICD therapy were not significant in our sub- group analysis.

Conflicting data exist on the prognostic significance of programmed ventricular stimulation during electrophysiological study in patients with ARVC [4,9,26]. Our subgroup analysis with five eligible studies concluded that inducibility during electrophysiological study can be a significant predictor of ventricular tachyarrhythmias in patients with ARVC. Nonetheless, it should be borne in mind that there surely exists a limitation of electrophysiological studies in arrhythmic risk stratification in general, and that current guidelines do not support the routine use of programmed ventricular stimulations for risk stratification in ARVC [16,27].

Many individual studies have suggested that asymptomatic patients with nonsustained ventricular tachyarrhythmias show a somewhat high (3-6\%/year) appropriate ICD discharge rate and significant (2\%/year) ventricular fibrillation rate [8,9]. In line with the findings from individual studies, nonsustained ventricular tachyarrhythmias on 24-hour Holter monitoring were identified as a useful independent predictor of ventricular tachyarrhythmias in our subgroup analysis. However, frequent ventricular ectopy on 24-hour Holter monitoring in asymptomatic patients did not predict the occurrence of ventricular tachyarrhythmias, despite a previous suggestion that an increased ventricular ectopy burden may be associated with electrical instability leading to malignant ventricular tachyarrhythmias over time [9].

Syncope is one of the most typical clinical presentations in adolescents or young individuals with ARVC [27]. A history of syncope as a predictive factor for ventricular tachyarrhythmias in ARVC has been evaluated by many groups [9,28-30]; however, a clear conclusion has not been reached yet. The significance of syncope as a risk factor of lethal tachyarrhythmias in patients with ARVC was first reported by Marcus et al. [30]. Although a previous study has even suggested that patients with prior syncope have a four-fold increased risk for subsequent ventricular tachyarrhythmias [8], the result of our subgroup analysis suggests that prior syncope cannot predict subsequent ventricular tachyarrhythmias. Syncope itself may be a nonspecific clinical feature, may not be of an arrhythmic origin, and may be linked to a neurocardiogenic mechanism. An inclusion of nonarrhythmic syncope would possibly make the differential diagnosis difficult and its prognostic value elusive. Nevertheless, a previous study by Bhonsale et al. observed that significantly more patients 
with recent (less than 6 months) unexplained syncope received ICD therapy than those with remote syncope [9], which can be considered in clinical situations.

There has been one study that evaluated and speculated that the proband status might be a factor for risk prediction of ventricular tachyarrhythmias in ARVC; 36 probands (90\%) had appropriate ICD therapies compared with only four family members (9\%) who received ICD therapy [9]. More extensive analyses may be needed on this issue.

In summary, ICD therapy is mandatory for patients with ARVC with previous cardiac arrest or sustained ventricular tachyarrhythmias. For the primary prophylaxis of sudden cardiac events, this meta-analysis demonstrated that extensive right ventricular dysfunction, ventricular late potential on SAECG, inducible ventricular tachyarrhythmias during electrophysiologic study, and non-sustained ventricular tachyarrhythmias are meaningful risk factors of sudden death in patients with ARVC. However, the following are the limitations of our analysis: (1) most of the studies included were relatively small retrospective observational studies, and (2) an inevitable potential duplicate patient inclusion may exist. As such, we cannot recommend the application of our result automatically in the consideration of primary ICD implantation; deciding primary ICD implantation should remain as an individual decision based on whether any of the above risk factors are present.

\section{CONFLICT OF INTEREST}

No potential conflict of interest relevant to this article was reported.

\section{ORCID}

Young-Eun Roh, https://orcid.org/0000-0001-7348-2758

Min-Jung Cho, https://orcid.org/0000-0002-6884-853X

\section{REFERENCES}

1. Thiene G, Nava A, Corrado D, Rossi L, Pennelli N. Right ventricular cardiomyopathy and sudden death in young people. N Engl J Med 1988;318:129-33.

2. Corrado D, Basso C, Rizzoli G, Schiavon M, Thiene G. Does sports activity enhance the risk of sudden death in adolescents and young adults? J Am Coll Cardiol 2003;42:1959-63.

3. Holst AG, Winkel BG, Theilade J, Kristensen IB, Thomsen JL, Ottesen GL, et al. Incidence and etiology of sports-related sudden cardiac death in Denmark--implications for preparticipation screening. Heart Rhythm 2010;7:1365-71.

4. Corrado D, Leoni L, Link MS, Della Bella P, Gaita F, Curnis A, et al. Implantable cardioverter-defibrillator therapy for prevention of sudden death in patients with arrhythmogenic right ventricular cardiomyopathy/dysplasia. Circulation 2003; 108:3084-91.

5. Wichter T, Paul M, Wollmann C, Acil T, Gerdes P, Ashraf $\mathrm{O}$, et al. Implantable cardioverter/defibrillator therapy in arrhythmogenic right ventricular cardiomyopathy: single-center experience of long-term follow-up and complications in 60 patients. Circulation 2004;109:1503-8.

6. Piccini JP, Dalal D, Roguin A, Bomma C, Cheng A, Prakasa $\mathrm{K}$, et al. Predictors of appropriate implantable defibrillator therapies in patients with arrhythmogenic right ventricular dysplasia. Heart Rhythm 2005;2:1188-94.

7. Pezawas T, Stix G, Kastner J, Schneider B, Wolzt M, Schmidinger $H$. Ventricular tachycardia in arrhythmogenic right ventricular dysplasia/cardiomyopathy: clinical presentation, risk stratification and results of long-term follow-up. Int J Cardiol 2006;107:360-8.

8. Corrado D, Calkins H, Link MS, Leoni L, Favale S, Bevilacqua $\mathrm{M}$, et al. Prophylactic implantable defibrillator in patients with arrhythmogenic right ventricular cardiomyopathy/ dysplasia and no prior ventricular fibrillation or sustained ventricular tachycardia. Circulation 2010;122:1144-52.

9. Bhonsale A, James CA, Tichnell C, Murray B, Gagarin D, Philips B, et al. Incidence and predictors of implantable cardioverter-defibrillator therapy in patients with arrhythmogenic right ventricular dysplasia/cardiomyopathy undergoing implantable cardioverter-defibrillator implantation for primary prevention. J Am Coll Cardiol 2011;58:1485-96.

10. Schuler PK, Haegeli LM, Saguner AM, Wolber T, Tanner FC, Jenni R, et al. Predictors of appropriate ICD therapy in patients with arrhythmogenic right ventricular cardiomyopathy: long term experience of a tertiary care center. PLoS One 2012;7:e39584.

11. Battipaglia I, Scalone G, Macchione A, Pinnacchio G, Laurito M, Milo M, et al. Association of heart rate variability with arrhythmic events in patients with arrhythmogenic right ventricular cardiomyopathy/dysplasia. Circ J 2012;76:618-23.

12. Santangeli P, Dello Russo A, Pieroni M, Casella M, Di Biase L, Burkhardt JD, et al. Fragmented and delayed electrograms within fibrofatty scar predict arrhythmic events in arrhythmogenic right ventricular cardiomyopathy: results from a prospective risk stratification study. Heart Rhythm 2012;9: 1200-6.

13. Canpolat U, Kabakçi G, Aytemir K, Dural M, Sahiner L, Yorgun $\mathrm{H}$, et al. Fragmented QRS complex predicts the arrhythmic events in patients with arrhythmogenic right ventricular cardiomyopathy/dysplasia. J Cardiovasc Electrophysiol 2013; 24:1260-6.

14. Link MS, Laidlaw D, Polonsky B, Zareba W, McNitt S, Gear 
$\mathrm{K}$, et al. Ventricular arrhythmias in the North American multidisciplinary study of ARVC: predictors, characteristics, and treatment. J Am Coll Cardiol 2014;64:119-25.

15. Ruiz-Salas A, Cabrera-Bueno F, García-Pinilla JM, BarreraCordero A, Peña-Hernández J, Fernández-Pastor J, et al. Longterm prognosis of patients with arrhythmogenic right ventricular cardiomyopathy and implantable defibrillator. Int J Cardiol 2014;174: 794-6.

16. Zipes DP, Camm AJ, Borggrefe M, Buxton AE, Chaitman B, Fromer M, et al. Guidelines for management of patients with ventricular arrhythmias and the prevention of sudden cardiac death. Executive summary. Rev Esp Cardiol 2006;59: 1328.

17. Chung FP, Li HR, Chong E, Pan CH, Lin YJ, Chang SL, et al. Seasonal variation in the frequency of sudden cardiac death and ventricular tachyarrhythmia in patients with arrhythmogenic right ventricular dysplasia/cardiomyopathy: the effect of meteorological factors. Heart Rhythm 2013;10: 1859-66.

18. Roguin A, Bomma CS, Nasir K, Tandri H, Tichnell C, James $\mathrm{C}$, et al. Implantable cardioverter-defibrillators in patients with arrhythmogenic right ventricular dysplasia/cardiomyopathy. J Am Coll Cardiol 2004;43:1843-52.

19. Marcus FI, Fontaine GH, Guiraudon G, Frank R, Laurenceau JL, Malergue C, et al. Right ventricular dysplasia: a report of 24 adult cases. Circulation 1982;65:384-98.

20. Sen-Chowdhry S, Lowe MD, Sporton SC, McKenna WJ. Arrhythmogenic right ventricular cardiomyopathy: clinical presentation, diagnosis, and management. Am J Med 2004;117: 685-95.

21. Sen-Chowdhry S, Prasad SK, Syrris P, Wage R, Ward D, Merrifield $\mathrm{R}$, et al. Cardiovascular magnetic resonance in arrhythmogenic right ventricular cardiomyopathy revisited: comparison with task force criteria and genotype. J Am Coll Cardiol 2006;48:2132-40.
22. Gemayel C, Pelliccia A, Thompson PD. Arrhythmogenic right ventricular cardiomyopathy. J Am Coll Cardiol 2001;38: 1773-81.

23. Blomström-Lundqvist C, Olsson SB, Edvardsson N. Followup by repeated signal-averaged surface QRS in patients with the syndrome of arrhythmogenic right ventricular dysplasia. Eur Heart J 1989;10(Suppl D):54-60.

24. Turrini P, Angelini A, Thiene G, Buja G, Daliento L, Rizzoli $\mathrm{G}$, et al. Late potentials and ventricular arrhythmias in arrhythmogenic right ventricular cardiomyopathy. Am J Cardiol 1999;83:1214-9.

25. Migliore F, Zorzi A, Michieli P, Perazzolo Marra M, Siciliano M, Rigato I, et al. Prevalence of cardiomyopathy in Italian asymptomatic children with electrocardiographic T-wave inversion at preparticipation screening. Circulation 2012;125: 529-38.

26. Wichter T, Paul M, Wollmann C, Acil T, Gerdes P, Ashraf $\mathrm{O}$, et al. Implantable cardioverter/defibrillator therapy in arrhythmogenic right ventricular cardiomyopathy: single-center experience of long-term follow-up and complications in 60 patients. Circulation 2004;109:1503-8.

27. Basso C, Corrado D, Bauce B, Thiene G. Arrhythmogenic right ventricular cardiomyopathy. Circ Arrhythm Electrophysiol 2012;5:1233-46.

28. Hulot JS, Jouven X, Empana JP, Frank R, Fontaine G. Natural history and risk stratification of arrhythmogenic right ventricular dysplasia/cardiomyopathy. Circulation 2004;110: 1879-84.

29. Dalal D, Nasir K, Bomma C, Prakasa K, Tandri H, Piccini $\mathrm{J}$, et al. Arrhythmogenic right ventricular dysplasia: a United States experience. Circulation 2005;112:3823-32.

30. Marcus FI, Fontaine GH, Frank R, Gallagher JJ, Reiter MJ. Long-term follow-up in patients with arrhythmogenic right ventricular disease. Eur Heart J 1989;10(Suppl D):68-73. 